\title{
Front Matter: Volume 6922
}

, "Front Matter: Volume 6922," Proc. SPIE 6922, Metrology, Inspection, and Process Control for Microlithography XXII, 692201 (22 April 2008); doi: $10.1117 / 12.798984$

SPIE. Event: SPIE Advanced Lithography, 2008, San Jose, California, United States 


\section{PROCEEDINGS OF SPIE}

\section{Metrology, Inspection, and Process Control for Microlithography XXII}

John A. Allgair

Christopher J. Raymond

Editors

25-28 February 2008

San Jose, California, USA

Sponsored and Published by

SPIE

Cooperating Organization

SEMATECH (USA)

Volume 6922

Part One of Two Parts 
The papers included in this volume were part of the technical conference cited on the cover and title page. Papers were selected and subject to review by the editors and conference program committee. Some conference presentations may not be available for publication. The papers published in these proceedings reflect the work and thoughts of the authors and are published herein as submitted. The publisher is not responsible for the validity of the information or for any outcomes resulting from reliance thereon.

Please use the following format to cite material from this book:

Author(s), "Title of Paper," in Metrology, Inspection, and Process Control for Microlithography XXII, edited by John A. Allgair, Christopher J. Raymond, Proceedings of SPIE Vol. 6922 (SPIE, Bellingham, WA, 2008) Article CID Number.

ISSN 0277-786X

ISBN 9780819471079

Published by

SPIE

P.O. Box 10, Bellingham, Washington $98227-0010$ USA

Telephone +1 3606763290 (Pacific Time) · Fax +1 3606471445

SPIE.org

Copyright (c) 2008, Society of Photo-Optical Instrumentation Engineers

Copying of material in this book for internal or personal use, or for the internal or personal use of specific clients, beyond the fair use provisions granted by the U.S. Copyright Law is authorized by SPIE subject to payment of copying fees. The Transactional Reporting Service base fee for this volume is $\$ 18.00$ per article (or portion thereof), which should be paid directly to the Copyright Clearance Center (CCC), 222 Rosewood Drive, Danvers, MA 01923. Payment may also be made electronically through CCC Online at copyright.com. Other copying for republication, resale, advertising or promotion, or any form of systematic or multiple reproduction of any material in this book is prohibited except with permission in writing from the publisher. The CCC fee code is $0277-786 \mathrm{X} / 08 / \$ 18.00$.

Printed in the United States of America.

Publication of record for individual papers is online in the SPIE Digital Library.

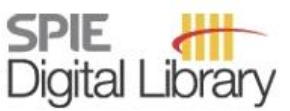

SPIEDigitalLibrary.org

Paper Numbering: Proceedings of SPIE follow an e-First publication model, with papers published first online and then in print and on CD-ROM. Papers are published as they are submitted and meet publication criteria. A unique, consistent, permanent citation identifier (CID) number is assigned to each article at the time of the first publication. Utilization of CIDs allows articles to be fully citable as soon they are published online, and connects the same identifier to all online, print, and electronic versions of the publication. SPIE uses a six-digit CID article numbering system in which:

- The first four digits correspond to the SPIE volume number.

- The last two digits indicate publication order within the volume using a Base 36 numbering system employing both numerals and letters. These two-number sets start with $00,01,02,03,04,05$, 06, 07, 08, 09, OA, OB ... 0Z, followed by 10-1Z, 20-2Z, etc.

The CID number appears on each page of the manuscript. The complete citation is used on the first page, and an abbreviated version on subsequent pages. Numbers in the index correspond to the last two digits of the six-digit CID number. 


\title{
Contents
}

\section{Part One}

\author{
xvii Symposium Committee \\ xix Conference Committee
}

\section{SESSION 1 INVITED SESSION}

692202 Diffraction order control in overlay metrology: a review of the roadmap options (Invited Paper) [6922-01]

M. Adel, D. Kandel, V. Levinski, J. Seligson, A. Kuniavsky, KLA-Tencor Corp. (Israel)

692203 Overlay metrology at the crossroads (Invited Paper) [6922-02]

N. P. Smith, Nanometrics Taiwan (Taiwan); L. A. Binns, Nanometrics, Ltd. (United Kingdom);

A. Plambeck, K. Heidrich, Nanometrics, Inc. (USA)

\section{SESSION 2 SOLUTIONS FOR TODAY}

692204 Production aspects of $45 \mathrm{~nm}$ immersion lithography defect monitoring using laser DUV inspection methodology [6922-03]

R. Kirsch, A. Martin, U. Okoroanyanwu, W. Grundkeb, U. Vogler, AMD (Germany); M. Beyer, Applied Materials Europe (Germany); E. Valfer, Applied Materials Israel (Israel); S. WeiherTellford, Applied Materials Europe (Germany); R. Perlovitch, N. Racah, Applied Materials Israel (Israel); P. Vanoppen, R. Moerman, ASML (Netherlands)

692206 Immersion lithography bevel solutions [6922-158]

L. Tedeschi, O. Tamada, M. Sanada, S. Yasuda, M. Asai, SOKUDO Co., Ltd. (Japan)

692207 Versatile DUV scatterometer of the PTB and FEM based analysis for mask metrology [6922-05]

M. Wurm, A. Diener, B. Bodermann, H. Gross, R. Model, Physikalisch-Technische Bundesanstalt (Germany); A. Rathsfeld, Weierstraß-Institut für Angewandte Analysis und Stochastik Mohrenstr (Germany)

692208 Toward accurate feature shape metrology [6922-06]

N. G. Orji, R. G. Dixson, National Institute of Standards and Technology (USA); B. D. Bunday, J. A. Allgair, International SEMATECH Manufacturing Initiative (USA)

692209 Extracting dose and focus from critical dimension data: optimizing the inverse solution [6922-07]

K. R. Lensing, J. B. Stirton, S. Chauhan, Advanced Micro Devices, Inc. (USA) 
6922 OA Challenges of implementing contour modeling in 32nm technology [6922-08]

D. Fischer, G. Han, J. Oberschmidt, IBM Systems and Technology Group (USA); Y. W. Cheng, Chartered Semiconductor Manufacturing (USA); J. Y. Maeng, Samsung Electronics Co. (USA); C. Archie, W. LU, IBM Systems and Technology Group (USA); C. Tabery, Advanced Micro Devices (USA)

\section{SESSION 3 METHODS FOR TOMORROW}

6922 OB The potentials of helium ion microscopy for semiconductor process metrology [6922-09] M. T. Postek, A. E. Vladár, National Institute of Standards and Technology (USA)

6922 OC Evaluating diffraction based overlay metrology for double patterning technologies [6922-10]

C. S. Saravanan, Y. Liu, P. Dasari, Nanometrics, Inc. (USA); O. Kritsun, C. Volkman, A. Acheta, B. La Fontaine, Advanced Micro Devices, Inc. (USA)

6922 OD Plasma cleaning of nanoparticles from EUV mask materials by electrostatics [6922-12] W. M. Lytle, R. Raju, H. Shin, C. Das, M. J. Neumann, D. N. Ruzic, Univ. of Illinois at UrbanaChampaign (USA)

6922 OE Optical through-focus technique that differentiates small changes in line width, line height, and sidewall angle for CD, overlay, and defect metrology applications [6922-13] R. Attota, R. Silver, National Institute of Standards and Technology (USA); B. M. Barnes, KT Consulting, Inc. (USA)

\section{SESSION 4 STANDARDS AND REFERENCE METROLOGY}

6922 OF Paving the way for multiple applications for the 3D-AFM technique in the semiconductor industry [6922-14]

J. Foucher, CEA-LETI-MINATEC (France); E. Pargon, M. Martin, LTM (France); S. Reyne, CEALETI-MINATEC (France); C. Dupré, CEA-LETI-MINATEC (France) and IMEP-LAHC, INPGMINATEC (France)

6922 OG Controlled deposition of NIST-traceable nanoparticles as additional size standards for photomask applications [6922-15]

J. Wang, D. Y. H. Pui, C. Qi, Univ. of Minnesota (USA); S.-J. Yook, Hanyang Univ.

(South Korea); H. Fissan, Institute of Energy and Environmental Technology e.V. (Germany); E. Ultanir, T. Liang, Intel Corp. (USA)

$6922 \mathrm{OH} \quad$ Accurate and traceable dimensional metrology with a reference CD-SEM [6922-16] A. E. Vladár, J. S. Villarrubia, P. Cizmar, M. Oral, M. T. Postek, National Institute of Standards and Technology (USA)

6922 Ol Sub-nanometer pitch calibration and data quality evaluation methodology [6922-17] C.-M. Ke, Y. Wang, J. Huang, J. Hu, J. Huang, T.-S. Gau, B. J. Lin, Taiwan Semiconductor Manufacturing Co. (Taiwan)

6922 0J A novel AFM method for sidewall measurement of high-aspect ratio patterns [6922-18] M. Watanabe, S. Baba, T. Nakata, Hitachi, Ltd. (Japan); T. Morimoto, S. Sekino, Hitachi Kenki FineTech Co., Ltd. (Japan) 
6922 OK TEM validation of CD AFM image reconstruction: part II [6922-19]

G. A. Dahlen, Thorleaf Research, Inc. (USA); H.-C. Liu, M. Osborn, J. R. Osborne, Veeco

Instruments, Inc. (USA); B. Tracy, A. del Rosario, Spansion, Inc. (USA)

$6922 \mathrm{OL}$ Dimension controlled CNT probe of AFM metrology tool for 45-nm node and beyond [6922-20]

S. Sekino, T. Morimoto, T. Kurenuma, Hitachi Kenki FineTech Co., Ltd. (Japan); M. Hirooka, Hitachi, Ltd. (Japan); H. Tanaka, Hitachi Kyowa Engineering Co., Ltd. (Japan)

\section{SESSION 5 OVERLAY I}

6922 OM Overlay metrology tool calibration using blossom [6922-21]

L. A. Binns, N. P. Smith, P. Dasari, Nanometrics, Inc. (USA)

$69220 \mathrm{~N} \quad$ Using in-chip overlay metrology [6922-22]

S. Girol-Gunia, B. Schulz, AMD Fab 36 LLC and Co. KG (Germany); N. Smith, L. Binns, Nanometrics, Inc. (United Kingdom)

692200 Diffraction based overlay metrology: accuracy and performance on front end stack [6922-23]

P. Leray, S. Cheng, IMEC (Belgium); D. Kandel, M. Adel, A. Marchelli, I. Vakshtein, KLATencor Corp. (Israel); M. Vasconi, STMicroelectronics (Italy); B. Salski, QWED (Poland)

$6922 \mathrm{OP}$ Optimization of high order control including overlay, alignment, and sampling [6922-106] D. Choi, KLA-Tencor (South Korea); C. Lee, C. Bang, D. Cho, M. Gil, Hynix Semiconductor, Inc. (South Korea); P. Izikson, KLA-Tencor (Israel); S. Yoon, D. Lee, KLA-Tencor (South Korea)

$69220 Q \quad$ Overlay measurement based on dual-overlay grating image [6922-24]

D.-M. Shyu, Y. Ku, S.-P. Dong, Industrial Technology Research Institute (Taiwan)

\section{SESSION 6 SCATTEROMETRY I}

6922 OR Assessing scatterometry for measuring advanced spacer structures with embedded SiGe [6922-25] M. Sendelbach, S. Zangooie, IBM Corp. (USA); A. Vaid, Advanced Micro Devices, Inc. (USA); P. Herrera, J. Leng, I. Kim, KLA-Tencor Corp. (USA)

6922 OS Characterization of $32 \mathrm{~nm}$ node BEOL grating structures using scatterometry [6922-26] S. Zangooie, M. Sendelbach, M. Angyal, C. Archie, IBM Microelectronics (USA); A. Vaid, I. Matthew, Advanced Micro Devices Inc. (USA); P. Herrera, KLA-Tencor Corp. (USA)

6922 OT Advanced profile control and the impact of sidewall angle at gate etch for critical nodes [6922-27]

H. Lee, A. Ranjan, D. Prager, Tokyo Electron America, Inc. (USA); K. A. Bandy, E. Meyette, IBM Microelectronics (USA); R. Sundararajan, A. Viswanathan, A. Yamashita, M. Funk, Tokyo Electron America, Inc. (USA) 
6922 OU Scatterometry as technology enabler for embedded SiGe process [6922-28]

A. Vaid, R. Pal, Advanced Micro Devices, Inc. (USA); M. Sendelbach, S. Zangooie, IBM Corp. (USA); K. Lensing, Advanced Micro Devices, Inc. (USA); C. Hartig, AMD Saxony Manufacturing GmbH (Germany)

6922 OV Measurement of high-k and metal film thickness on FinfET sidewalls using scatterometry [6922-29]

T. G. Dziura, KLA-Tencor (USA); B. Bunday, International SEMATECH Manufacturing Initiative (USA); C. Smith, M. M. Hussain, R. Harris, SEMATECH (USA); X. Zhang, KLA-Tencor (USA); J. M. Price, SEMATECH (USA)

$6922 \mathrm{OW}$ Industrial characterization of scatterometry for advanced APC of $65 \mathrm{~nm}$ CMOS logic gate patterning [6922-30]

K. Dabertrand, M. Touchet, STMicroelectronics (France); S. Kremer, KLA-Tencor (France);

C. Chaton, CEA-LETI-Minatec (France); M. Gatefait, E. Aparicio, STMicroelectronics

(France); M. Polli, KLA-Tencor (France); J.-C. Royer, CEA-LETI-Minatec (France)

\section{SESSION 7 PROCESS CONTROL}

6922 0X Impact of sampling on uncertainty: semiconductor dimensional metrology applications [6922-31]

B. Bunday, ISMI, CD Metrology (USA); B. Rijpers, ASML, Research and Development, CD Metrology (Netherlands); B. Banke, C. Archie, IBM Microelectronics (USA); I. B. Peterson, Applied Materials, Inc. (USA); V. Ukraintsev, Veeco Instruments, Inc. (USA); T. Hingst, Qimonda Dresden (USA); M. Asano, Toshiba Corp. (Japan)

6922 OY CD uniformity control via real-time control of photoresist properties [6922-32] M. Chen, J. Fu, W. K. Ho, A. Tay, National Univ. of Singapore (Singapore)

$69220 Z$ Process control for $\mathbf{4 5} \mathbf{n m}$ CMOS logic gate patterning [6922-33] B. Le Gratiet, P. Gouraud, E. Aparicio, L. Babaud, K. Dabertrand, M. Touchet, STMicroelectronics (France); S. Kremer, KLA-Tencor (France); C. Chaton, CEA-Leti (France); F. Foussadier, F. Sundermann, J. Massin, J.-D. Chapon, M. Gatefait, B. Minghetti, J. de-Caunes, D. Boutin, STMicroelectronics (France)

692210 Improvement of gate CD uniformity for $\mathbf{5 5} \mathbf{~ n m}$ node logic devices [6922-34] T. Murakami, T. Nakata, K. Taniguchi, T. Uchiyama, NEC Electronics Corp. (Japan); M. Jyousaka, M. Tadokoro, Tokyo Electron Kyushu, Ltd. (Japan); Y. Konishi, Tokyo Electron Software Technologies, Ltd. (Japan)

$692211 \quad$ Metrology characterization for self-aligned double patterning [6922-35]

A. Berger, S. Latinsky, M. Bar-Zvi, R. Peltinov, Applied Materials (Israel); J. Shu, C. Ngai, J. Yu, H. Dai, Applied Materials (USA)

692212 Focus and dose control to actual process wafer [6922-36]

H. Ina, K. Sentoku, Canon, Inc. (Japan) 
692213 Defect criticality index (DCI): a new methodology to significantly improve DOI sampling rate in a 45nm production environment [6922-37]

Y. Sato, Y. Yamada, Y. Kaga, Y. Yamazaki, Toshiba Corp. (Japan); M. Aoki, D. Tsui, C. Young, E. Chang, KLA-Tencor Corp. (USA)

692214 Lot acceptance sampling inspection plan for non-normal CD distribution [6922-38] T. Ikeda, M. Asano, Toshiba Corp. (Japan)

692215 Improvements on the simulation of microscopic images for the defect detection of nanostructures [6922-39]

S. Rafler, T. Schuster, K. Frenner, W. Osten, Institut für Technische Optik (Germany);

U. Seifert, Qimonda Dresden GmbH and Co. OHG (Germany)

692216 Defect inspection using a high-resolution pattern image obtained from multiple lowresolution images of the same pattern on an observed noisy SEM image [6922-40] M. Takashima, Y. Midoh, K. Nakamae, Osaka Univ. (Japan)

692217 Contamination specification for dimensional metrology SEMs [6922-41]

A. E. Vladár, K. P. Purushotham, M. T. Postek, National Institute of Standards and Technology (USA)

692218 In-line inspection resistance mapping using quantitative measurement of voltage contrast in SEM images [6922-42]

M. Matsui, Y. Anan, T. Odaka, Hitachi, Ltd. (Japan); H. Nagaishi, K. Sakurai, Renesas Technology Corp. (Japan)

\section{SESSION 9 CDSEM}

692219 Experiment and simulation of charging effects in SEM [6922-43]

S. Babin, S. Borisov, aBeam Technologies, Inc. (USA); Y. Miyano, H. Abe, M. Kadowaki,

A. Hamaguchi, Y. Yamazaki, Toshiba Corp. (Japan)

6922 1A Characterization of CD-SEM metrology for iArF photoresist materials [6922-44]

B. Bunday, A. Cordes, International SEMATECH Manufacturing Initiative (USA); N. G. Orji, National Institute of Standards and Technology (USA); E. Piscani, D. Cochran, J. Byers, SEMATECH, Inc. (USA); J. Allgair, International SEMATECH Manufacturing Initiative (USA); B. J. Rice, SEMATECH, Inc. (USA); Y. Avitan, R. Peltinov, M. Bar-zvi, O. Adan, Applied Materials (Israel)

6922 1B Advanced CD-SEM metrology to improve total process control performance for hyper-NA lithography [6922-45] M. Osaki, Hitachi, Ltd. (Japan) and IMEC vzw (Belgium); M. Tanaka, C. Shishido, Hitachi, Ltd. (Japan); T. Ishimoto, Hitachi High-Technologies Corp. (Belgium) and IMEC vzW (Belgium); N. Hasegawa, Hitachi High-Technologies Corp. (Japan); K. Sekiguchi, Hitachi HighTechnologies Europe GmbH (Germany) and IMEC vzw (Belgium); K. Watanabe, Hitachi High-Technologies Corp. (Japan); S. Cheng, D. Laidler, M. Ercken, E. Altamirano, IMEC (Belgium) 
6922 IC CD-SEM contour-based process monitoring in DRAM production environment [6922-47] U. Kramer, D. Jackisch, R. Wildfeuer, S. Fuchs, F. Jauzion-Graverolle, Qimonda Dresden GmbH and Co. OHG (Germany); G. Ben-Nahumb, O. Menadeva, S. Ventola, Applied Materials, Inc. (Israel)

\section{SESSION 10 OVERLAY II}

6922 1D Accurate in-resolution level overlay metrology for multipatterning lithography techniques [6922-48]

I. Englard, R. Piech, C. Masia, Applied Materials Europe (Netherlands); N. Hillel, L. Gershtein, D. Sofer, R. Peltinov, O. Adan, Applied Materials (Israel)

$69221 \mathrm{E} \quad$ Sources of overlay error in double patterning integration schemes [6922-49]

D. Laidler, P. Leray, K. D'havé, S. Cheng, IMEC (Belgium)

6922 IF Correlating overlay metrology precision to interlayer dielectric film properties [6922-52] K. R. Paserba, Seagate Technology LLC (USA)

6922 1G Overlay improvement by zone alignment strategy [6922-53]

C.-Y. Huang, A.-Y. Lee, C.-L. Shih, Nanya Technology Corp. (Taiwan); R. Yang, M. Yuan,

H. Chen, R. Chang, Inotera Memories, Inc. (Taiwan)

\section{SESSION 11 CD FOR DEVELOPMENT AND OPC}

$69221 \mathrm{H} \quad$ Challenges of OPC model calibration from SEM contours [6922-54]

Y. Granik, I. Kusnadi, Mentor Graphics Corp. (USA)

$692211 \quad$ Empirical data validation for model building [6922-55]

A. Kazarian, Synopsys, Inc. (USA)

$69221 \mathrm{~J}$ Automated creation of production metrology recipes based on design information [6922-56]

J. P. Cain, M. Threefoot, K. Shah, Advanced Micro Devices, Inc. (USA); B. Schulz, S. GirolGunia, J.-T. Hoeft, AMD Fab36 LLC and Co. KG (Germany)

$69221 \mathrm{~K}$ Impact of assistance feature to pattern profile for isolated feature in sub-65 nm node [6922-57]

M. Kim, Y.-J. Yun, E. Jeong, K. Choi, J. Kim, J. Han, DongbuHitek Co., Ltd. (South Korea)

$69221 \mathrm{~L}$ Accurate device simulations through CD-SEM-based edge-contour extraction [6922-95]

E. Shauly, Tower Semiconductors (Israel); O. Menadeva, Applied Materials, Inc. (Israel);

R. Drori, M. Cohen-Yasour, I. Rotstein, Tower Semiconductors (Israel); R. Peltinov, A. Bartov, S. Latinski, A. Siany, M. Geshesl, Applied Materials, Inc. (Israel) 
$69221 \mathrm{M} \quad$ Angle resolved optical metrology [6922-59]

R. M. Silver, National Institute of Standards and Technology (USA); B. M. Barnes, KT Consulting, Inc. (USA); A. Heckert, R. Attota, R. Dixson, National Institute of Standards and Technology (USA); J. Jun, KT Consulting, Inc. (USA)

$69221 \mathrm{~N}$ Opportunities and challenges for optical CD metrology in double patterning process control [6922-60]

D. C. Wack, J. Hench, L. Poslavsky, J. Fielden, V. Zhuang, W. Mieher, T. Dziura, KLA-Tencor (USA)

692210 Forward solve algorithms for optical critical dimension metrology [6922-61]

P. L. Jiang, H. Chu, Tokyo Electron, Ltd. (USA); J. Hench, D. Wack, KLA-Tencor (USA)

6922 IP Comparison of spectroscopic Mueller polarimetry, standard scatterometry, and real space imaging techniques (SEM and 3D-AFM) for dimensional characterization of periodic structures [6922-62]

A. De Martino, M. Foldyna, T. Novikova, Lab. de Physique des Interfaces et des Couches Minces, Ecole Polytechnique (France); D. Cattelan, Jobin Yvon HORIBA (France);

P. Barritault, C. Licitra, J. Hazart, J. Foucher, F. Bogeat, CEA-LETI Minatec (France)

\section{SESSION 13 CDSEM II}

6922 IR Physical matching of CD-SEM: noise analysis and verification in FAB environment [6922-65] U. Kramer, A. Navarra, G. Fleischer, J. Kaiser, F. Voss, Qimonda Dresden GmbH and Co. OHG (Germany); G. Zuckerman, R. Kris, I. Ben-Dayan, E. Sommer, A. Len, S. Dror, Applied Materials, Inc. (Israel); D. Schöne, S. Ventola, Applied Materials, Inc. (Germany)

6922 is AWV: high-throughput cross-array cross-wafer variation mapping [6922-66] J.-H. Yeo, B.-H. Lee, T.-Y. Lee, Samsung Electronics Co., Ltd. (South Korea); G. Greenberg, D. Meshulach, E. Ravid, S. Levi, K. Kan, S. Shabtay, Y. Cohen, O. Rotlevy, Applied Materials Israel (Israel)

6922 IT CD bias reduction in CD-SEM linewidth measurements for advanced lithography [6922-67] M. Tanaka, Hitachi, Ltd. (Japan); J. Meessen, ASML (Netherlands); C. Shishido, Hitachi, Ltd. (Japan); K. Watanabe, Hitachi High-Technologies Corp. (Japan); I. Minnaert-Janssen, P. Vanoppen, ASML (Netherlands)

$69221 \mathrm{U}$ Automatic CD-SEM offline recipe creation in a high volume production fab [6922-69] S. Girol-Gunia, S. Roling, AMD Fab36 LLC and Co. KG (Germany); O. Menadeva, D. Levitzky, A. Costa, D. Fischer, Applied Materials, Inc. (Israel)

6922 IV Automated CD-SEM metrology for efficient TD and HVM [6922-70]

A. Starikov, S. P. Mulapudi, Intel Corp. (USA) 


\section{Part Two}

\section{SESSION 14 NOVEL METHODS AND APPLICATIONS}

6922 IW Modeling for metrology with a helium beam [6922-71]

R. Ramachandra, Univ. of Tennessee (USA); B. J. Griffin, Univ. of Western Australia (Australia) and Oak Ridge National Lab. (USA); D. C. Joy, Univ. of Tennessee (USA) and Oak Ridge National Lab. (USA)

$69221 \mathrm{X}$ Novel CD inspection technology leveraging a form birefringence in a Fourier space [6922-72]

A. Kawai, D. Mochida, Nikon Corp. (Japan); K. Yoshino, Y. Yamazaki, Toshiba Corp. (Japan)

$69221 \mathrm{Y}$ Experimental quantification of reticle electrostatic damage below the threshold for ESD [6922-73]

G. C. Rider, Microtome, Inc. (USA); T. S. Kalkur, Univ. of Colorado at Colorado Springs (USA)

$69221 \mathrm{Z}$ Linewidth roughness and cross-sectional measurements of sub-50 nm structures with CDSAXS and CD-SEM [6922-74]

C. Wang, National Institute of Standards and Technology (USA); K.-W. Choi, Intel Corp. (USA); R. L. Jones, C. Soles, E. K. Lin, W. Wu, National Institute of Standards and Technology (USA); J. S. Clarke, Intel Corp. (USA); J. S. Villarrubia, National Institute of Standards and Technology (USA); B. Bunday, International SEMATECH Manufacturing Initiative (USA)

\section{SESSION 15 LINE-EDGE ROUGHNESS}

692220 A novel method for pushing the limits of line edge roughness detection by scatterometry [6922-75]

Y. Cohen, Nova Measuring Instruments Ltd. (Israel); B. Yaakobovitz, Y. Tsur, Technion-Israel Institute of Technology (Israel); D. Scheiner, Nova measuring Instruments, Ltd. (Israel)

692221 Influence of image processing on line-edge roughness in CD-SEM measurement [6922-76] A. Yamaguchi, J. Yamamoto, Hitachi, Ltd. (Japan)

692222 Practical and bias-free LWR measurement by CDSEM [6922-77] S.-B. Wang, Y. H. Chiu, H. J. Tao, Y. J. Mii, Taiwan Semiconductor Manufacturing Co., Ltd. (Taiwan)

692223 Fractal dimension of line width roughness and its effects on transistor performance [6922-156]

V. Constantoudis, E. Gogolides, Institute of Microelectronics (Greece)

\section{POSTER SESSION}

692224 Exploring the limitations of x-ray reflectivity as a critical dimension pattern shape metrology [6922-11]

H.-J. Lee, S. Kim, C. L. Soles, E. K. Lin, W. Wu, National Institute of Standards and Technology (USA) 
692225 Probe-pattern grating focus monitor through scatterometry calibration [6922-63]

J. Xue, C. J. Spanos, A. R. Neureuther, Univ. of California, Berkeley (USA)

692226 An objective image focus for CD-SEM [6922-68]

H. Zhang, C. Gould, B. Roberts, M. McQuillan, Qimonda North America (USA)

692227 Ellipsometric inspection of the inner surface of pellicle-covered masks [6922-78]

S. Lee, Hanyang Univ. (South Korea); C. Song, Hanyang Univ. (South Korea) and Samsung Electronics Co. (South Korea); J. Rhim, H. Lee, J. Kyoung, Hanyang Univ. (South Korea);

S. Chin, T. Ahn, Samsung Electronics Co. (South Korea); I. An, Hanyang Univ. (South Korea)

692228 Optics characterization with compact EUV spectrophotometer [6922-79]

H. Blaschke, I. Balasa, L. Koch, K. Starke, D. Ristau, Laser Zentrum Hannover e.V. (Germany);

C. Wies, R. Lebert, AIXUV Aachen (Germany); A. Bayer, F. Barkusky, K. Mann, Laser-Lab.

Göttingen e.V. (Germany)

692229 Verification of optics for the die-to-wafer-like image mask inspection [6922-80]

A. Takada, T. Tojo, Topcon Corp. (Japan); M. Shibuya, Tokyo Polytechnic Univ. (Japan)

6922 2A Phase metrology on 45-nm node phase-shift mask structures [6922-81]

K. M. Lee, M. Tavassoli, Intel Mask Operation (USA); U. Buttgereit, D. Seidel, R. Birkner,

S. Perlitz, Carl Zeiss SMS GmbH (Germany)

6922 2B A new high-resolution photomask inspection system for contamination detection [6922-82] B. Mu, A. Dayal, L.-H. Yiin, J. Zhu, J. Miller, G. Inderhees, KLA-Tencor Corp. (USA)

6922 2C Systematic defect inspection and verification for distributions of critical dimension in OPC models utilizing design based metrology tool [6922-83]

J.-G. Park, S. Lee, Y.-S. Kang, Y.-K. Park, Samsung Electronics Co. Ltd. (South Korea);

T. Kitamura, T. Hasebe, S. Nakazawa, NanoGeometry Research Inc. (Japan)

6922 2D Advanced method to monitor design-process marginality for $65 \mathrm{~nm}$ node and beyond [6922-84]

C. Huang, Advanced Metrology Dept. CRD Advanced Modules Div. (USA); C. Young, KLATencor Corp. (USA); H. Liu, S. F. Tzou, Advanced Metrology Dept. CRD Advanced Modules Div. (USA); D. Tsui, E. Chang, KLA-Tencor Corp. (USA)

6922 2E CD-SAXS measurements using laboratory-based and synchrotron-based instruments [6922-85]

C. Wang, National Institute of Standards and Technology (USA); K.-W. Choi, Intel Corp. (USA); W.-E. Fu, D. L. Ho, R. L. Jones, C. Soles, E. K. Lin, W. Wu, National Institute of Standards and Technology (USA); J. S. Clarke, Intel Corp. (USA); B. Bunday, International SEMATECH Manufacturing Initiative (USA)

6922 2F A novel methodology for model-based OPC verification [6922-86]

T. Huang, C. Liao, Nanya Technology Corp. (Taiwan); R. Chou, H.-Y. Liao, J. Schacht, Mentor Graphics Corp. (Taiwan)

6922 2H Effect of setpoint on CD measurement in CD-AFM: plausibility study [6922-88]

B. C. Park, J. Choi, S. J. Ahn, Korea Research Institute of Standards and Science

(South Korea); M. Shin, D. Ihm, B. Lee, Samsung Electronics Co., Ltd. (South Korea) 
6922 2J Recent CD AFM probe developments for sub-45 nm technology nodes [6922-91]

H.-C. Liu, J. R. Osborne, G. A. Dahlen, Veeco Instruments, Inc. (USA); J. Greschner, T. Bayer, S. Kalt, G. Fritz, Team Nanotec GmbH (Germany)

6922 2K Electron-beam-patterning simulation and metrology of complex layouts on Si/Mo multilayer substrates [6922-92]

G. P. Patsis, D. Drygiannakis, Institute of Microelectronics (Greece); N. Tsikrikas, Institute of Microelectronics (Greece) and National Technical Univ. of Athens (Greece); I. Raptis,

E. Gogolides, Institute of Microelectronics (Greece)

$69222 \mathrm{~L} \quad$ Application of model-based library approach to $\mathrm{Si}_{3} \mathrm{~N}_{4}$ hardmask measurements [6922-93] M. Tanaka, C. Shishido, W. Nagatomo, Hitachi, Ltd. (Japan); K. Watanabe, Hitachi HighTechnologies Corp. (Japan)

6922 2M Calibration of CD-SEM: moving from relative to absolute measurements [6922-94] S. Babin, S. Borisov, A. Ivanchikov, I. Ruzavin, Abeam Technologies, Inc. (USA)

$69222 \mathrm{~N} \quad$ Automated metrology for SEM calibration and CD line measurements using image analysis and SEM modeling methods [6922-96]

V. Khvatkov, V. Alievski, R. Kadushnikov, Smart Imaging Technologies (USA); S. Babin, aBeam Technologies (USA)

692220 Further study on the verification of CD-SEM based monitoring for hyper NA lithography [6922-97]

T. Ishimoto, Hitachi High-Technologies Corp. (Japan); M. Osaki, Hitachi, Ltd. (Japan);

K. Sekiguchi, Hitachi High-Technologies Europe GmbH (Germany); N. Hasegawa,

K. Watanabe, Hitachi High-Technologies Corp. (Japan); D. Laidler, S. Cheng, IMEC vzw (Belgium)

6922 2P MUGFET observation and CD measurement by using CD-SEM [6922-98]

T. Maeda, Hitachi High-Technologies Corp. (Japan); M. Tanaka, Hitachi, Ltd. (Japan);

M. Isawa, K. Watanabe, N. Hasegawa, Hitachi High-Technologies Corp. (Japan);

K. Sekiguchi, Hitachi High-Technologies Europe GmbH (Germany); R. Rooyackers,

N. Collaert, T. Vandeweyer, IMEC vzw (Belgium)

$69222 \mathrm{Q} \quad$ High order correction and sampling strategy for $45 \mathrm{~nm}$ immersion lithography overlay control [6922-99]

B. Y. Hsueh, G. K. C. Huang, C.-C. Yu, J. K. C. Hsu, United Microelectronics Corp. (Taiwan); C.-C. K. Huang, C.-J. Huang, D. Tien, KLA-Tencor Corp. (USA)

6922 2R Improve overlay control and scanner utilization through high order corrections [6922-100] H. M. Lin, B. Lin, J. Wu, S. Chiu, Powerchip Semiconductor Corp. (Taiwan); C.-C. K. Huang, J. Manka, D. Goh, H. Huang, D. Tien, KLA-Tencor Corp. (USA)

692225 Overlay control using scatterometry based metrology (SCOM) in production environment [6922-101]

B. Dinu, KLA-Tencor Corp. (Israel); S. Fuchs, U. Kramer, M. Kubis, Qimonda Dresden GmbH and Co. OHG (Germany); A. Marchelli, KLA-Tencor Corp. (Israel); A. Navarra, Qimonda Dresden GmbH and Co. OHG (Germany); C. Sparka, A. Widmann, KLA-Tencor Corp. (Israel) 
6922 2T Alignment system and process optimization for improvement of double patterning overlay [6922-103]

W. Ma, J. Kang, C. Lim, H. Kim, S. Moon, Hynix Semiconductor, Inc. (South Korea);

S. Lalbahadoersing, S. Oh, ASML (Netherlands)

$69222 \mathrm{U}$ Sampling for advanced overlay process control [6922-105]

D. Choi, KLA-Tencor Korea Co., Ltd. (South Korea); P. Izikson, KLA-Tencor Corp. (Israel);

D. Sutherland, K. Sherman, J. Manka, J. C. Robinson, KLA-Tencor Corp. (USA)

6922 2V A system to optimize mix-and-match overlay in lithography [6922-107]

S. Wakamoto, Y. Ishii, K. Yasukawa, Nikon Corp. (Japan); S. Maejima, Renesas Technology Corp. (Japan); A. Kato, KLA-Tencor Japan, Ltd. (Japan); J. C. Robinson, KLA-Tencor Corp. (USA); D.-S. Choi, KLA-Tencor Korea Co., Ltd. (South Korea)

6922 2W Diffraction based overlay metrology for $\alpha$-carbon applications [6922-108]

C. S. Saravanan, A. Tan, P. Dasari, G. Goelzer, N. Smith, Nanometrics Inc. (USA); S.-H. Woo, J. H. Shin, H. J. Kang, H. C. Kim, Samsung Electronics Co., Ltd. (South Korea)

6922 2X Film stacking architecture for immersion lithography process [6922-109] T. Goto, M. Sanada, T. Miyagi, K. Shigemori, M. Kanaoka, S. Yasuda, O. Tamada, M. Asai, SOKUDO Co., Ltd. (Japan)

6922 2Y Controlling macro and micro surface topography for a $45 \mathrm{~nm}$ copper CMP process using a high resolution profiler [6922-111]

T. Ortleb, G. Marxsen, J. Heinrich, AMD LLC and Co. KG (Germany); J. Reichert, R. Haupt, P. Yam, KLA-Tencor (USA)

$69222 Z$ Effects produced by CDU improvement of resist pattern with PEB temperature control for wiring resistance variation reduction [6922-112]

M. Tadokoro, S. Shinozuka, Tokyo Electron Kyushu, Ltd. (Japan); K. Ogata, T. Morimoto, Tokyo Electron, Ltd. (Japan)

692230 Rationalizing the mechanism of HMDS degradation in air and effective control of the reaction byproducts [6922-113]

K. Seguin, A. J. Dallas, G. Weineck, Donaldson Co., Inc. (USA)

692231 Stress measurement system for process control [6922-114]

K. Akashika, M. Horie, Dainippon Screen Manufacturing Co., Ltd. (Japan)

692234 CDU improvement technology of etching pattern using photo lithography [6922-117] M. Tadokoro, S. Shinozuka, M. Jyousaka, Tokyo Electron Kyushu, Ltd. (Japan); K. Ogata, T. Morimoto, Tokyo Electron, Ltd. (Japan); Y. Konishi, Tokyo Electron Software Technologies, Ltd. (Japan)

692235 Film thickness measurement tool with a stress measurement function [6922-118] M. Horie, K. Akashika, S. Shiota, S. Yamaguchi, K. Yamano, Dainippon Screen Manufacturing Co., Ltd. (Japan) 
692237 In-situ real-time temperature control of baking systems in lithography [6922-120] Y. Wang, National Univ. of Singapore (Singapore); H.-T. Chua, The Univ. of Western Australia (Australia); A. Tay, National Univ. of Singapore (Singapore)

692238 Dimensionality reduction methods in virtual metrology [6922-121]

D. Zeng, Y. Tan, C. J. Spanos, Univ. of California, Berkeley (USA)

692239 Wide applications of design based metrology with tool integration [6922-123]

H. Yang, J. Kim, A. Jung, T. Lee, D. Yim, J. Kim, Hynix Semiconductor, Inc. (South Korea);

T. Hasebe, M. Yamamoto, NanoGeometry Research, Inc. (Japan)

6922 3A Wafer edge polishing process for defect reduction during immersion lithography [6922-125]

M. Okazaki, Applied Materials (USA); R. Maas, ASML Netherlands B.V. (Netherlands);

S.-H. Ko, Y. Chen, P. Miller, M. Thothadri, M. Dutta, C.-P. Chang, A. Anapolsky, C. Lazik,

Y. Uritsky, M. Seamons, D. Padhi, W. Yeh, Applied Materials (USA); S. Sinkwitz, ASML

Netherlands B.V. (Netherlands); C. Ngai, Applied Materials (USA)

6922 3B High throughput wafer defect monitor for integrated metrology applications in photolithography [6922-126]

N. Rao, P. Kinney, A. Gupta, Real Time Metrology, Inc. (USA)

6922 3D UV-reflectometory for fast trench-depth measurement [6922-128]

M. Horie, S. Shiota, S. Yamaguchi, K. Yamano, M. Kobayashi, Dainippon Screen

Manufacturing Co., Ltd. (Japan)

6922 3E Study of ADI (After Develop Inspection) on photo resist wafers using electron beam (III): novel method for ADI on metal hard mask by penetration contrast [6922-129]

T. Hayashi, M. Saito, K. Fujihara, Tokyo Electron, Ltd. (Japan); J. Jau, Hermes Microvision, Inc. (USA)

$69223 G \quad$ Improving dry etch control for contact plugs in advanced DRAM manufacturing [6922-131] T. Bao, Veeco Instruments, Inc. (USA); Y. Bar, Micron Technology, Inc. (USA); D. Fong, Veeco Instruments, Inc. (USA); M. Godbole, Micron Technology, Inc. (USA)

$69223 \mathrm{H} \quad$ In-line focus-dose monitoring for hyper NA imaging [6922-132]

S. Loi, A. Fasciszewski Zeballos, U. lessi, STMicroelectronics (Italy); J. Robinson, KLA-Tencor

Corp. (USA); P. Izikson, KLA-Tencor (Israel); A. Mani, M. Polli, KLA-Tencor (Italy)

6922 3J Picometer-scale accuracy in pitch metrology by optical diffraction and atomic force microscopy [6922-134]

D. A. Chernoff, Advanced Surface Microscopy, Inc. (USA); E. Buhr, Physikalisch-Technische Bundesanstalt (Germany); D. L. Burkhead, Advanced Surface Microscopy, Inc. (USA);

A. Diener, Physikalisch-Technische Bundesanstalt (Germany)

6922 3K Development of back-end-of-the-line applications using optical digital profilometry (ODP) [6922-135]

J.-J. Huang, J. H. Yeh, United Microelectronics Corp. (Taiwan); Y. Luo, L. Wu, Y. Wen, Timbre Technologies (USA)

6922 3L Scatterometry based overlay metrology [6922-136]

T. Matsumoto, H. Ina, K. Sentoku, S. Oishi, Canon, Inc. (Japan) 
$69223 \mathrm{M}$ Spectroscopic ellipsometer for ultra thin film [6922-137]

K. Akashika, S. Shiota, S. Yamaguchi, M. Horie, M. Kobayashi, Dainippon Screen

Manufacturing Co., Ltd. (Japan)

$69223 \mathrm{~N}$ Characterization of sub-50-nm line array structures with angle-resolved multiple wavelength scatterometry [6922-139]

M. Kotelyanskii, F. Shen, G. Jiang, Rudolph Technologies, Inc. (USA); B. Bunday, ISMI (USA)

692230 Sensitivity and performance estimates for the multiple wavelength multiple incidence angle ellipsometry for OCD applications [6922-140]

M. Kotelyanskii, G. Jiang, Rudolph Technologies, Inc. (USA)

$69223 \mathrm{P} \quad$ Modeling the effect of finite size gratings on scatterometry measurements [6922-142]

E. Kenyon, M. W. Cresswell, National Institute of Standards and Technology (USA);

H. J. Patrick, National Institute of Standards and Technology (USA) and KT Consulting, Inc.

(USA); T. A. Germer, National Institute of Standards and Technology (USA)

$69223 \mathrm{Q}$ Characterization of the poly gate $\mathrm{ACl}$ structure with laser based angle resolved multiple wavelength scatterometry [6922-143]

G. Jiang, M. Kotelyanskii, F. Shen, Rudolph Technologies, Inc. (USA)

6922 3R Low-k n\&k variation impact on CD accuracy of scatterometry [6922-145]

Y. Chen, Timbre Technologies, Inc. (USA); M. Yamamoto, Tokyo Electron, Ltd. (Japan);

D. Likhachev, G. He, Timbre Technologies, Inc. (USA); A. Sonoda, Tokyo Electron, Ltd.

(Japan); V. Vuong, Timbre Technologies, Inc. (USA)

692235 Implementation of spectroscopic critical dimension (SCD) for leveling inline monitor of ASML 193nm scanner [6922-146]

W. K. Lin, M. Yeh, United Microelectronics Corp. (Taiwan)

6922 3T 3D semiconductor grooves measurement simulations (scatterometry) using nonstandard FDTD methods [6922-147]

H. Shirasaki, Tamagawa Univ. (Japan)

$69223 \mathrm{U}$ Novel approach for immersion lithography defectivity control to increase productivity [6922-148]

I. Englard, Applied Materials Europe (Netherlands); R. Stegen, P. Vanoppen,

I. Minnaert-Janssen, T. der Kinderen, E. van Brederode, F. Duray, J. Linders, D. Ovchinnikov, ASML (Netherlands); R. Piech, C. Masia, Applied Materials Europe (Netherlands); N. Hillel,

E. Ravid, O. Rotlevi, A. Wilde, S. Shabtay, Z. Telor, Applied Materials Israel (Israel);

R. Schreutelkamp, Applied Materials Europe (Netherlands)

$69223 \mathrm{~V}$ Traceable calibration of AFM step height measurements for integrated circuit manufacturing [6922-149]

J. Robert, B. Banke, IBM Microelectronics (USA); R. Dixson, National Institute of Standards and Technology (USA); C. Strocchia-Rivera, IBM Microelectronics (USA)

$69223 X \quad 22 \mathrm{~nm}$ node contact hole formation in extreme ultra-violet lithography [6922-151] E.-J. Kim, Hanyang Univ. (South Korea); K.-H. Kim, H.-R. Park, J.-Y. Yeo, J.-S. Kim, Seoul National Univ. (South Korea); H.-K. Oh, Hanyang Univ. (South Korea) 
$69223 Y$ Advanced lithography parameters extraction by using scatterometry system: part II [6922-152]

W. Zhou, M. Hsieh, H. Koh, M. Zhou, Chartered Semiconductor Manufacturing, Ltd. (Singapore)

692242 Compensating for SSIS sizing/classification error in a defect review SEM world [6922-157] D. Ruprecht, MEMC Electronic Materials, Inc. (USA); S. McGarvey, Hitachi High Technologies America, Inc. (USA)

Author Index 


\title{
Symposium Committees
}

\author{
Symposium Chair
}

Roxann L. Engelstad, University of Wisconsin, Madison (USA)

Symposium Cochair

Christopher J. Progler, Photronics, Inc. (USA)

Technical Organizing Committees

Executive Committee

Robert D. Allen, IBM Almaden Research Center (USA)

John A. Allgair, SEMATECH, Inc. (USA) and Advanced Micro

Devices, Inc. (USA)

Mircea Dusa, ASML US, Inc. (USA)

Roxann L. Engelstad, University of Wisconsin, Madison (USA)

Clifford L. Henderson, Georgia Institute of Technology (USA)

Bruno LaFontaine, Advanced Micro Devices, Inc. (USA)

Harry J. Levinson, Advanced Micro Devices, Inc. (USA)

Christopher J. Progler, Photronics, Inc. (USA)

Christopher J. Raymond, Nanometrics, Inc. (USA)

Michael L. Rieger, Synopsys, Inc. (USA)

Franklin M. Schellenberg, Mentor Graphics Corporation (USA)

Vivek K. Singh, Intel Corporation (USA)

Advisory Committee

Robert D. Allen, IBM Almaden Research Center (USA)

William H. Arnold, ASML (USA)

Timothy A. Brunner, IBM Thomas J. Watson Research Center (USA)

Ralph R. Dammel, AZ Electronic Materials USA Corporation (USA)

Roderick R. Kunz, MIT Lincoln Laboratory (USA)

Harry J. Levinson, Advanced Micro Devices, Inc. (USA)

Burn Lin, Taiwan Semiconductor Manufacturing Company, Ltd. (Taiwan)

Chris A. Mack, LithoGuru.com (USA)

Victor Pol, Freescale Semiconductor, Inc. (USA)

Michael T. Postek, National Institute of Standards and Technology (USA)

Luc Van den Hove, IMEC (Belgium)

C. Grant Willson, The University of Texas at Austin (USA)

Anthony Yen, Taiwan Semiconductor Manufacturing Company, Ltd. (Taiwan) and Cymer, Inc. (USA) 
Downloaded From: https://www.spiedigitallibrary.org/conference-proceedings-of-spie on 25 Apr 2023

Terms of Use: https://www.spiedigitallibrary.org/terms-of-use 


\title{
Conference Committee
}

\author{
Conference Chair
}

John A. Allgair, SEMATECH, Inc. (USA) and Advanced Micro Devices, Inc. (USA)

Conference Cochair

Christopher J. Raymond, Nanometrics, Inc. (USA)

Program Committee

Ofer Adan, Applied Materials (Israel)

Michael J. Anderson, Rohm and Haas Electronic Materials (USA)

Charles N. Archie, IBM Corporation (USA)

Jason P. Cain, Advanced Micro Devices, Inc. (USA)

Alain G. Deleporte, STMicroelectronics (France)

Daniel J. C. Herr, Semiconductor Research Corporation (USA)

David C. Joy, The University of Tennessee (USA)

Chih-Ming Ke, Taiwan Semiconductor Manufacturing Company, Ltd. (Taiwan)

Byoung-Ho Lee, SAMSUNG Electronics Company, Ltd. (South Korea)

Martha I. Sanchez, IBM Almaden Research Center (USA)

Richard M. Silver, National Institute of Standards and Technology (USA)

Bhanwar Singh, Advanced Micro Devices, Inc. (USA)

Alexander Starikov, Intel Corporation (USA)

Neal T. Sullivan, Arradiance, Inc. (USA)

Brian M. Trafas, KLA-Tencor Corporation (USA)

Vladimir A. Ukraintsev, Veeco Instruments, Inc. (USA)

\section{Session Chairs}

1 Invited Session

John A. Allgair, SEMATECH, Inc. (USA) and Advanced Micro Devices, Inc. (USA)

Christopher J. Raymond, Nanometrics Inc. (USA)

2 Solutions for Today

Brian M. Trafas, KLA-Tencor Corporation (USA)

Alexander Starikov, Intel Corporation (USA) 
3 Methods for Tomorrow

Christopher J. Raymond, Nanometrics, Inc. (USA)

Daniel J. C. Herr, Semiconductor Research Corporation (USA)

$4 \quad$ Standards and Reference Metrology

Vladimir A. Ukraintsev, Veeco Instruments, Inc. (USA)

David C. Joy, The University of Tennessee (USA)

5 Overlay I

Brian M. Trafas, KLA-Tencor Corporation (USA)

Daniel J. C. Herr, Semiconductor Research Corporation (USA)

6 Scatterometry I

Christopher J. Raymond, Nanometrics, Inc. (USA)

Richard M. Silver, National Institute of Standards and Technology (USA)

7 Process Control

Jason P. Cain, Advanced Micro Devices, Inc. (USA)

Alexander Starikov, Intel Corporation (USA)

8 Inspection and Defect

Byoung-Ho Lee, SAMSUNG Electronics Company, Ltd. (South Korea)

Martha I. Sanchez, IBM Almaden Research Center (USA)

9 CDSEM I

John A. Allgair, SEMATECH, Inc. (USA) and Advanced Micro Devices, Inc. (USA)

Ofer Adan, Applied Materials (Israel)

10 Overlay II

David C. Joy, The University of Tennessee (USA)

11 CD for Development and OPC

Ofer Adan, Applied Materials (Israel)

Christopher J. Raymond, Nanometrics, Inc. (USA)

12 Scatterometry II

Christopher J. Raymond, Nanometrics, Inc. (USA)

Alexander Starikov, Intel Corporation (USA)

13 CDSEM II

Martha I. Sanchez, IBM Almaden Research Center (USA)

John A. Allgair, SEMATECH, Inc. (USA) and Advanced Micro Devices, Inc. (USA) 
$14 \quad$ Novel Methods and Applications

Richard M. Silver, National Institute of Standards and Technology (USA)

Jason P. Cain, Advanced Micro Devices, Inc. (USA)

15 Line-Edge Roughness

Martha I. Sanchez, IBM Almaden Research Center (USA)

Ofer Adan, Applied Materials (Israel) 
Downloaded From: https://www.spiedigitallibrary.org/conference-proceedings-of-spie on 25 Apr 2023

Terms of Use: https://www.spiedigitallibrary.org/terms-of-use 